\title{
PROBLEMS IN STRONG UNIFORM DISTRIBUTION
}

\author{
KWO CHAN — RADHAKRISHNAN NAIR
}

\begin{abstract}
In $1923 \mathrm{~A}$. Khinchin asked if given any $B \subseteq[0,1)$ of positive Lebesgue measure, we have $\frac{1}{N} \#\{n: 1 \leq n \leq N:\{n x\} \in B\} \rightarrow|B|$ for almost all $x$ with respect to Lebesgue measure. Here $\{y\}$ denotes the fractional part of the real number $y$ and $|A|$ denotes the Lebesgue measure of the set $A$ in $[0,1)$. In $1970 \mathrm{~J}$. Marstrand showed the answer is no. In this paper the authors survey contributions to this subject since then.
\end{abstract}

\section{Introduction}

The fact that for an irrational number $\alpha$ the sequence $(\{n \alpha\})_{n \geq 1}$ is dense in $[0,1)$ is ascribed to $\mathrm{L}$. K r o n e c k e r, though essentially the same observation was made by $\mathrm{N}$. Ores me in the fourteenth century.

Following H. W e yl [27] we say that a sequence $\left(x_{n}\right)_{n \geq 1}$ is uniformly distributed modulo 1 (u.d. mod 1) if for each interval $I$ (closed on the left and open on the right) of length $|I|$, we have

$$
\lim _{N \rightarrow \infty} \frac{1}{N} \sum_{n=1}^{N} \chi_{I}\left(\left\{x_{n}\right\}\right)=|I| .
$$

Here $\chi_{I}(x)=1$ if $x \in I$ and $\chi_{I}(x)=0$ if $x \notin I$. We call $\chi_{I}$ the characteristic function of $I$.

It was proved by P. B ohl [3], W. Sierpinski [24] and H. W eyl [26] that if $\alpha$ is irrational and if $x_{n}=n \alpha(n=1,2, \ldots)$, then the sequence $\left(x_{n}\right)_{n>1}$ is u.d. mod 1. In 1914 and $1916 \mathrm{H}$. W e y l [26, 27] gave the following famous and extremely useful characterization of uniform distribution.

THEOREM 1. The following are equivalent

(i) $\left(x_{n}\right)_{n \geq 1}$ is u.d. $\bmod 1$;

(ii) $\frac{1}{N} \sum_{n=1}^{N} f\left(\left\{x_{n}\right\}\right) \rightarrow \int_{0}^{1} f(t) d t$ for $f \in C([0,1))$

(continuous functions on $[0,1)$ );

(c) 2014 Mathematical Institute, Slovak Academy of Sciences.

2010 Mathematics Subject Classification: 11K55.

Keywords: strong uniform distribution, ergodic averages. 
(iii) $\frac{1}{N} \sum_{n=1}^{N} f\left(\left\{x_{n}\right\}\right) \rightarrow \int_{0}^{1} f(t) d t$ for $f \in R([0,1))$

(Riemann integrable functions on $[0,1)$ );

and

(iv) $\frac{1}{N} \sum_{n=1}^{N} e^{2 \pi i h x_{n}} \rightarrow 0$ for all $h \in \mathbb{Z} \backslash\{0\}$

(Weyl's criterion).

Some applications due to $H$. W e y l [27]:

1) $\frac{1}{N}\left|\sum_{n=1}^{N} e^{2 \pi i h n \alpha}\right| \leq 2 \frac{1}{N}\left|e^{2 \pi i h \alpha}-1\right|^{-1}$, hence if $\alpha \notin \mathbb{Q}$, and $x_{n}=n \alpha$, then $\left(x_{n}\right)_{n \geq 1}$ is u.d. $\bmod 1$.

2) More generally using "differencing", if $p(x)=\alpha_{0}+\alpha_{1} x+\cdots+\alpha_{k} x^{x}$ and $\left(\alpha_{1}, \ldots, \alpha_{k}\right) \notin \mathbb{Q}^{k}$, then for $x_{n}=p(n)$ the sequence $\left(x_{n}\right)_{n \geq 1}$ is u.d. $\bmod 1$.

$3)$ If $\left(a_{n}\right)_{n \geq 1}$ is a sequence of distinct natural numbers, then $\left(a_{n} x\right)_{n \geq 1}$ is u.d. $\bmod 1$ for almost all $x$ with respect to Lebesgue measure.

In light of application 1) in $1923 \mathrm{~A}$. K hinchin [8] asked if given any $B \subseteq[0,1)$ of positive Lebesgue measure, we have

$$
\frac{1}{N} \#\{n: 1 \leq n \leq N:\{n x\} \in B\} \rightarrow|B| \text { for almost all } x
$$

with respect to Lebesgue measure. This was disproved by J. M. M a r st r a nd [10] in the following theorem.

TheOREm 2. There exists a $G_{\delta}$ set $B \subseteq[0,1)$ such that

$$
\varlimsup \frac{1}{N} \sum_{n=1}^{N} \chi_{B}(\{n x\})=1,
$$

almost everywhere with respect to Lebesgue measure and

$$
\underline{\varliminf} \frac{1}{N} \sum_{n=1}^{N} \chi_{B}(\{n x\})=0,
$$

almost everywhere with respect to Lebesgue measure.

In light of application 3) one might now wonder if there is any strictly increasing sequence of integers $\left(a_{n}\right)_{n \geq 1}$ such that given any $B \subseteq[0,1)$ of positive Lebesgue measure, we have

$$
\frac{1}{N} \#\left\{n: 1 \leq n \leq N:\left\{a_{n} x\right\} \in B\right\} \rightarrow|B|
$$

for almost all $x$ with respect to Lebesgue measure. To go further we need to discuss ergodic theory [25]. Let $(X, \beta, \mu)$ denote a probability space and let $T: X \rightarrow X$ denote a measurable (i.e., $T^{-1} A=\{x: T x \in A\}$ for all $A \in \beta$ ) 


\section{PROBLEMS IN STRONG UNIFORM DISTRIBUTION}

measure preserving transformation (i.e., $\mu\left(T^{-1}(A)\right)=\mu(A)$ for all $A \in \beta$ ) of a measure space. We say $(X, \beta, \mu, T)$ is ergodic if $\mu\left(A \Delta T^{-1} A\right)=0$ means $\mu(A) \in\{0,1\}$. Here for two sets $A$ and $B$ we have used $A \Delta B$ to denote their symmetric difference. We refer to the quadriple $(X, \beta, \mu, T)$ as a dynamical system. We first recall Birkhoff's pointwise ergodic theorem [25].

Theorem 3. Suppose the dynamical system $(X, \beta, \mu, T)$ is measurable and measure preserving. Then if

$$
f \in L^{1}(X, \beta, \mu),
$$

the limit

$$
\bar{f}(x)=\lim _{N \rightarrow \infty} \frac{1}{N} \sum_{n=0}^{N-1} f\left(T^{n} x\right),
$$

exists $\mu$ almost everywhere. Also $\bar{f}(T x)=\bar{f}(x) \mu$ almost everywhere. Further if $(X, \beta, \mu, T)$ is ergodic, then $\bar{f}(x)=\int_{X} f(t) d \mu \mu$ almost everywhere.

If $X=[0,1), \beta$ is the Lebesgue $\sigma$-algebra, $\mu$ is Lebesgue measure and $T x=\{p x\}$, where $p \in \mathbb{N} \backslash\{1\}$, we get the following classical special case called the Riesz-Raikov theorem [18], 20].

Theorem 4. If

$$
p \in \mathbb{N} \backslash\{1\} \quad \text { and } \quad f \in L^{1}([0,1)),
$$

then

$$
\frac{1}{N} \sum_{n=0}^{N-1} f\left(\left\{p^{n} x\right\}\right) \rightarrow \int_{0}^{1} f(t) d t \text { a.e., }
$$

w.r.t. Lebesgue measure.

If $X=[0,1), \beta$ is the Lebesgue $\sigma$-algebra, $\mu$ is Lebesgue measure and $T x=\{x+\alpha\}$, where $\alpha$ is irrational, then $T$ is Lebesgue measure preserving and we get the following theorem.

Theorem 5. If

$$
\alpha \notin \mathbb{Q} \quad \text { and } \quad f \in L^{1}([0,1)),
$$

then

$$
\frac{1}{N} \sum_{n=0}^{N-1} f(\{x+n \alpha\}) \rightarrow \int_{0}^{1} f(t) d t \text { a.e. }
$$

w.r.t. Lebesgue measure.

A natural question addressed by J. F. Koksma and R. Salem 9 is whether in light of application 3) of Weyl's criteria above the term $n \alpha$ in Theorem 5 can be replaced by $p(n)$. Some progress here is discussed in the next section. 


\section{KWO CHAN - RADHAKRISHNAN NAIR}

Using Theorem 4 J. M. Marstrand [10, proved the following.

THEOREM 6. If $\left(m_{k}\right)_{k>1}$ is a semi-group of integers generated multiplicatively by a finite set of co-prime natural numbers $\left\{p_{1}, \ldots, p_{l}\right\}$ all different from one and $f \in L^{\infty}([0,1))$, then

$$
\frac{1}{N} \sum_{k=1}^{N} f\left(\left\{m_{k} x\right\}\right) \rightarrow \int_{0}^{1} f(t) d t \text { a.e. }
$$

w.r.t. Lebesgue measure.

We call $\left(m_{k}\right)_{k \geq 1}$ the $l$-Hardy-Littlewood-Polya sequence (l-HLP sequence). Answering a question of R. C. B a k e r [1, using the general ergodic theorem described in Section 3, the second author [1] showed that $L^{\infty}$ can be replaced by $L^{1}$ in the above theorem. A much more complete resolution is the following.

Theorem 7. Suppose $A \subset \mathbb{N}$ and let $\left(m_{k}\right)_{k \geq 1}=\pi(A)$ denote the set of products of elements of $A$ ordered by absolute value.

(i) Then $\left(m_{k}\right)_{k \geq 1}$ is $\left(L^{1}\right)^{*}$ if and only if $\pi(A) \subseteq \pi(F)$ for a finite set $F$.

(ii) If $\left(m_{k}\right)_{k \geq 1}$ is not in $\pi(F)$ for any finite $F$, one can find a counter example of the form $f=\chi_{B}$, where $B$ is a $G_{\delta}$ set.

Theorem 7 (i) was proved by A. Quas and M. Wierdl using the same idea, which was used by the second author to answer Baker's question-namely using a general ergodic theorem. Theorem 7 (ii) appears in [6] as an application of Marstrand's method. A similar observation was made by G. K o z m a in unpublished work.

\section{The Koksma-Salem Problem}

Using harmonic analysis, J. F. Koksma and R. S a lem [9] showed the following

TheOrem 8. Suppose

$$
f \sim \sum_{n \in \mathbb{Z}} c_{n} e^{2 \pi i n x} \in L^{2}([0,1))
$$

Also suppose

$$
\sum_{|n| \geq N}\left|c_{n}\right|^{2}=O\left((\log N)^{-\gamma}\right) \quad \text { for } \gamma>0
$$

Then if

$$
p(x)=\alpha_{0}+\alpha_{1} x+\cdots+\alpha_{k} x^{k} \quad \text { with } \quad\left(\alpha_{1}, \ldots, \alpha_{k}\right) \notin \mathbb{Q}^{k},
$$

we have

$$
\lim _{N \rightarrow \infty} \frac{1}{N} \sum_{n=1}^{N} f(\{x+p(n)\})=\int_{0}^{1} f(t) d t,
$$

almost everywhere w.r.t Lebesgue measure. 


\section{PROBLEMS IN STRONG UNIFORM DISTRIBUTION}

In conversation with the second author of this paper, independently, both R. C. B aker and M. W eber asked if the decay condition on the coefficients $\left(c_{n}\right)_{n \in \mathbb{Z}}$ could be removed or weakened. The following progress is possible. Let $(X, \beta, \mu)$ be a probability space and let $T_{1}, \ldots, T_{l}$ denote commuting measurable, measure preserving transformation of $(X, \beta, \mu)$. J. B o u r g a in 4 claimed that if

$f \in L^{2}(X, \beta, \mu) \quad$ for $\quad l(x)=\lim _{N \rightarrow \infty} \frac{1}{N} \sum_{n=1}^{N} f\left(T_{1}^{n^{1}} \ldots T_{l}^{n^{l}} x\right) \quad$ exists a.e. w.r.t. $\mu$.

There seems, however, to the authors to be a non-trivial gap in the proof of this theorem. This gap has been filled in [17]. Further B o u r g a in [5] claims that $L^{2}$ can be replaced by $L^{p}$ for $p>1$. A detailed proof of this has never been published however. Using this assumption however, one can prove the result below 12 .

Let

$$
X=[0,1), \quad T_{1}(x)=\left\{x+\alpha_{1}\right\}, \ldots, T_{l}(x)=\left\{x+\alpha_{l}\right\} .
$$

Then if

$$
p(x)=\alpha_{0}+\alpha_{1} x+\cdots+\alpha_{l} x^{l} \quad \text { with } \quad\left(\alpha_{1}, \ldots, \alpha_{l}\right) \notin\left(\mathbb{Q}^{k}\right)^{c},
$$

note

$$
\frac{1}{N} \sum_{n=1}^{N} f(\{x+p(n)\})=\frac{1}{N} \sum_{n=1}^{N} f\left(T_{1}^{n^{1}} \ldots T_{l}^{n^{l}}\left(x+\alpha_{0}\right)\right) .
$$

Applying the above theorem and noting that $(p(n))_{n \geq 1}$ is u.d. mod 1, we must have $l(x)=\int_{0}^{1} f(t) d t$, i.e., if $f \in L^{p}([0,1))$ for $p>1$ we have

$$
\lim _{N \rightarrow \infty} \frac{1}{N} \sum_{n=1}^{N} f(\{x+p(n)\})=\int_{0}^{1} f(t) d t
$$

almost everywhere w.r.t. Lebesgue measure.

\section{Remarks about the proof of Theorem 7 (i)}

We begin by describing a general framework. Let $S$ be a countable abelian semigroup acting in a measure preserving fashion on a measure space $(\Omega, \mathcal{A}, \mu)$. That is, to each $g \in S$ there exists a measurable map $T_{g}$ of $\Omega$ such that $T_{g_{1}+g_{2}}=T_{g_{1}}\left(T_{g_{2}}\right)$ and for each $A$ in the $\sigma$-algebra $\mathcal{A}$ we have

$$
T_{g}^{-1} A=\left\{x \in \Omega: T_{g} x \in A\right\} \in \mathcal{A} \quad \text { for all } g \in S \quad \text { with } \quad \mu\left(T_{g}^{-1} A\right)=\mu(A) .
$$


Also let $\left(A_{k}\right)_{k=1}^{\infty}$ be a collection of subsets of $S$ such that the following conditions are true:

(B1) $0<\# A_{k}<\infty$;

(B2) $A_{k} \subset A_{k+1}$ for each $k \in \mathbf{N}$;

(B3) there exists a constant $M_{2}>0$ such that

$$
\#\left\{A_{k}-A_{k}\right\} \leq M_{2} \# A_{k}, \quad \text { for all } \quad k \in \mathbf{N} \text {; }
$$

(B4) (amenability)

$$
\lim _{k \rightarrow \infty} \frac{\#\left\{\left(g+A_{k}\right) \triangle A_{k}\right\}}{\# A_{k}}=0, \quad \text { for all } g \in S,
$$

where we have used $A \triangle B$ to denote the symmetric difference of the sets $A$ and $B$.

Here for a finite set $A$ we have used $\# A$ to denote its cardinality, and $A-A$ to denote $\{x \in S: y+x \in A$ for some $y \in A\}$.

From the above data we construct, the averages

$$
\pi_{k}(f)(x)=\frac{1}{\# A_{k}} \sum_{s \in A_{k}} T_{s} f(x) \quad \text { for } \quad k \in \mathbf{N},
$$

where $f \in L^{1}(\Omega, \mathcal{A}, \mu)$. We have a special case of T. B ew le y's theorem [2].

Theorem 9. Suppose (B1), (B2), (B3) and (B4) are true. Then

$$
\pi(f)(x)=\lim _{k \rightarrow \infty} \pi_{k}(f)(x)
$$

exists $\mu$ almost everywhere, with

$$
\int_{\Omega} \pi(f) d \mu=\int_{\Omega} f d \mu \text { and } T_{s}(\pi(f))(x)=\pi(f)(x)
$$

for almost all $x$, for each $s \in S$.

We further specialize this theorem as follows. Let $S=\prod_{n>1} \mathbb{N}$, i.e., the direct product of the natural numbers with themselves countably many times. The set $S$ may also be described as the space of sequences of elements of $\mathbb{N}$, all but finitely many of whose elements are non zero. To this semi-group $S$ we can associate an action of $S$ as follows. For a given sequence of integers $\left\{n_{1}, n_{2}, \ldots\right\}$ via the map $s \rightarrow T_{s}(s \in S)$ for $T_{s}:[0,1) \rightarrow[0,1)$, where if $s=\left(s_{1}, s_{2}, \ldots\right)$ we have $T_{s}(x)=\left\{n_{1}^{s_{1}} n_{2}^{s_{2}} \ldots x\right\}$. Notice that each non-identity element $T_{s}$ is ergodic and so $\pi(f)=\int_{0}^{1} f(t) d t$ almost everywhere with respect to Lebesgue measure [25]. We set

$$
A_{k}=\left\{n_{1}^{s_{1}} \ldots n_{l}^{s_{l}} \ldots \leq k: s=\left(s_{i}\right)_{i \geq 1} \in S\right\} .
$$

Clearly all the indefinite products $n_{1}^{s_{1}} \ldots n_{l}^{s_{l}} \ldots$ are actually finite and correspond to the individual natural numbers in the sequence $\left(m_{l}\right)_{l \geq 1}$. 


\section{PROBLEMS IN STRONG UNIFORM DISTRIBUTION}

It is immediate that (B1), (B2) and (B3) are satisfied by $\left(A_{k}\right)_{k \geq 1}$. To prove (B4) we have to show for each fixed $g \in S$, that

$$
\#\left\{\left(g+A_{k}\right) \backslash A_{k}\right\}, \#\left\{A_{k} \backslash\left(g+A_{k}\right)\right\}=o\left(\# A_{k}\right),
$$

as $k$ tends to infinity. Clearly this involves getting estimates for the numbers

$$
\text { \# }\left\{\left(g+A_{k}\right) \backslash A_{k}\right\}, \#\left\{A_{k} \backslash\left(g+A_{k}\right)\right\} \text { and } \# A_{k} .
$$

The authors know two methods for doing this. The first is the geometric approach of comparing the number of lattice points we are counting to the volumes of the regions they are in and estimating these volumes. The second is an inductive counting argument based on the number of generators at issue. In the case of $\mathbb{N}^{r}$ for finite $r \geq 1$ instead of $S$, these estimates are carried out using the first method in [1] and the second, in the second author's 1986 University Warwick $\mathrm{PhD}$. Extending these arguments to $S$, in the case, where $\left(m_{k}\right)_{k \geq 1}$ is contained in a finitely generated semigroup, is a very routine exercise using the fundamental theorem of arithmetic and so we forgo the details. The property (B4) follows. This proves Theorem 7 (i) once we observe that because $\pi(f)$.

\section{Remarks on the proof of Theorem 7 (ii)}

Our main tool is the following [10].

LEMма 10. Let $\left(m_{k}\right)_{k \geq 1}$ denote a strictly increasing sequence of integers. Suppose for each pair of integers $q, v>1$ that there exist pairs of sets of integers $G, H$ such that $(\mathrm{A}) \# G>v \# H$, and $(\mathrm{B})$ for every $g \in G$ there exists $\eta \geq 1$ such that $\mathrm{gm}_{k}^{-1} \in H$ for all $k \in[\eta, \eta q]$. Then there exists a $G_{\delta}$ set $B$ such that if $f=\chi_{B}$, the limit

$$
\lim _{N \rightarrow \infty} \frac{1}{N} \sum_{k=1}^{N} f\left(\left\{m_{k} \theta\right\}\right),
$$

fails to exist almost everywhere with respect to Lebesgue measure.

Using this lemma it is possible to prove that any sequence of natural numbers that is multiplicatively generated but not contained in a set of integers multiplicatively generated by any finite set must have the properties claimed for $\left(m_{k}\right)_{k \geq 1}$ by Lemma 10. This is now a well understood classical topic. See [10] for similar computations. Because of this rather than provide a detailed verification of this, we content ourselves with the following brief remark. 


\section{KWO CHAN — RADHAKRISHNAN NAIR}

Suppose $R$ is a large positive integer to be chosen at our convenience. Let

$$
M=\prod_{k=1}^{q R} m_{k}, \quad \text { and } \quad D=D(R, q)=p \max _{k \leq R} \frac{m_{q k}}{m_{k}},
$$

where $p$ is one of the primes dividing an element of $\left(m_{k}\right)_{k \geq 1}^{R}$ and let $P_{l}$ denote the semigroup of integers generated by the the first $l$ primes. Here $l$ is the smallest possible chosen so $P_{l}$ contains all the products in the set $\left(m_{k}\right)_{k=1}^{R}$. Now let $x$ be large and set

$$
H=[x, D x] \cap P_{l} \quad \text { with } \quad G=\left[m_{q} x, D m_{R}\right] \cap M P_{l},
$$

where $M P_{l}$ denotes the set of elements of $P_{l}$ multiplied by $M$. Following [10] we observe that

$$
\# H \sim K \log D(\log x)^{l-1} \text { and } \quad \# G \sim \frac{D m_{R}}{m_{q}}\left(\log \frac{x}{M}\right)^{s-1} .
$$

These two observations readily imply condition (A) of Lemma 10. To demonstrate (B) of Lemma 10 set $G_{\eta}=\left\{g \in M P_{l}: \frac{g}{m_{k}} \in H\right.$ for all $\left.r \in[\eta, q \eta]\right\}$ and note that $G_{\eta}=\left[m_{q \eta} x, m_{\eta} D x\right] \cap M P_{l}$. We can check that $\left[m_{q \eta} x, m_{\eta} D x\right]$ and $\left[m_{q(\eta+1)} x, m_{(\eta+1)} D x\right]$ intersect. Thus $G=\cup_{\eta=1}^{R} G_{\eta}$ as required. We summarise this in the following lemma.

\section{Marstrand's Lemma and its refinements}

To prove Theorem $6 \mathrm{M}$ arstrand proved the following important lemma.

THEOREM 11. Suppose strictly increasing sequences of natural numbers $a=\left(a_{r}\right)_{r=1}^{\infty}$ and $b=\left(b_{s}\right)_{s=1}^{\infty}$, are both $\left(L^{\infty}\right)^{*}$ sequences. Then the sequence generated multiplicatively by $a$ and $b$ once ordered by size is also an $\left(L^{\infty}\right)^{*}$ sequence. That is, for $f$ in $L^{\infty}([0,1))$ if

$$
G(u)=\left\{(r, s): a_{r} b_{s} \leq u\right\}
$$

we have

$$
\lim _{u \rightarrow \infty} \frac{1}{|G(u)|} \sum_{(r, s) \in G(u)} f\left(\left\{a_{r} b_{s} x\right\}\right)=\int_{0}^{1} f(t) d t
$$

almost everywhere with respect to Lebesgue measure.

Application: By the Riesz-Raikov theorem, a 1-HLP sequence is in $\left(L^{\infty}\right)^{*}$. Assume for the sake of induction that $a$ is an $(l-1)$-HLP sequences is in $\left(L^{\infty}\right)^{*}$. Also let $b$ be a 1-HLP sequence. Then $a \circ b=\left(a_{r} b_{s}\right)_{r, s \geq 1}$ ordered by absolute value is an $l$-HLP sequence and by Marstrand's lemma an $\left(L^{\infty}\right)^{*}$ sequence. This of course implies Theorem 6 . 


\section{PROBLEMS IN STRONG UNIFORM DISTRIBUTION}

Now we state an analogue of this Lemma for $L^{p}$ with finite $p>1$ due to the second author [13].

TheOREM 12. Suppose strictly increasing sequences of natural numbers

$$
a=\left(a_{r}\right)_{r=1}^{\infty} \text { and } b=\left(b_{s}\right)_{s=1}^{\infty},
$$

are both $\left(L^{p}\right)^{*}$ sequences for all $p>1$. Suppose also that there exists $C$ such that for each $u=1,2, \ldots$ we have

$$
\left|\left\{r: a_{r} \leq u\right\}\right|\left|\left\{s: b_{s} \leq u\right\}\right| \leq C\left|\left\{(r, s): a_{r} b_{s} \leq u\right\}\right| .
$$

Then the sequence generated multiplicatively by $a$ and $b$ once ordered by size is also an $\left(L^{p}\right)^{*}$ sequence for every $p>1$. That is, for $f$ in $L^{p}([0,1))$ for all $p>1$ if

$$
G(u)=\left\{(r, s): a_{r} b_{s} \leq u\right\},
$$

we have

$$
\lim _{u \rightarrow \infty} \frac{1}{|G(u)|} \sum_{(r, s) \in G(u)} f\left(\left\{a_{r} b_{s} x\right\}\right)=\int_{0}^{1} f(t) d t
$$

almost everywhere with respect to Lebesgue measure.

Let

$$
M f(x)=\sup _{N \geq 1}\left|\frac{1}{N} \sum_{n=1}^{N} f\left(\left\{a_{n} x\right\}\right)\right| .
$$

The idea is to use a theorem of S. S aw yer [23. This implies that a being an $\left(L^{p}\right)^{*}$ sequence for all $p>1$ is equivalent to the fact that for each $p$ there exists $C_{p}>0$ such that

$$
|\{x \in[0,1): M f(x) \geq \lambda\}| \leq \frac{C_{q}}{\lambda^{p}} \int_{0}^{1}|f| d t .
$$

Inequalities like this are called maximal equalities. The $L^{p}$ version of Marstrand's Lemma follows from iterating maximal inequalities. The following is a special case of the multi-parameter ergodic theorem used to prove Theorem 9, though it can be proved directly.

Suppose $(X, \beta, \mu, T)$ is a measurable, measure preserving dynamical system, $\phi: \mathbb{N} \rightarrow \mathbb{N}$ is a polynomial and $f \in L^{p}(X, \beta, \mu)$, then

$$
\lim _{N \rightarrow \infty} \frac{1}{N} \sum_{N=1}^{N} f\left(T^{\phi(n)} x\right), \quad \text { a.e. w.r.t. } \mu .
$$

The following is a special case.

Take $T x=\{p x\}(p>1)$ on $[0,1)$. Then $\left(p^{\phi(n)}\right)_{n \geq 1}$ is $\left(L^{p}\right)^{*}$. Using the $L^{p_{-}}$ Marstrand lemma, for $p_{1}, \ldots, p_{l} \in \mathbb{N} \backslash\{1\}$ and $\phi_{1}, \ldots, \phi_{l}$ all mapping $\mathbb{N}$ to itself, then $\left(m_{k}\right)_{k \geq 1}=\left(p_{1}^{\phi_{i}\left(n_{1}\right)} \ldots p_{l}^{\phi_{l}\left(n_{l}\right)}\right)_{\left(n_{1}, \ldots, n_{l}\right) \in \mathbb{N}^{l}}$ ordered by absolute value is in $\left(L^{p}\right)^{*}$ for $p \geq 1$. We have a further refinement [15]. 
TheOREM 13. Suppose $a_{1}=\left(a_{1, i}\right)_{i=1}^{\infty}, \ldots,\left(a_{k, i}\right)_{i=1}^{\infty}$ are $\left(L^{1}\right)^{*}$ sequences. Then if (11) holds with $a=a_{1}$ and $b=a_{2} \circ \ldots \circ a_{k}$ and $f$ belongs to $L\left(\log _{+} L\right)^{k-1}$,

$$
\lim _{u \rightarrow \infty} \frac{1}{|G(u)|} \sum_{c \in G(u)} f(\{c x\})=\int_{0}^{1} f(t) d t,
$$

almost everwhere with respect to Lebesgue measure.

\section{Another problem of R. C. Baker}

Another question of R. C. B a ker's [1] is whether there exists a sequence $\left(a_{n}\right)_{n \geq 1}$ which is not $\left(L^{\infty}\right)^{*}$ but for which it is true that

$$
\lim _{N \rightarrow \infty} \frac{1}{a_{N}} \sum_{j=0}^{a_{N}-1} f\left(\left\{x+\frac{j}{a_{N}}\right\}\right)=\int_{0}^{1} f(t) d t,
$$

almost everywhere with respect to Lebesgue measure holds.

In 1929 B. J es sen proved the following theorem [7].

THeOREM 14. For a strictly increasing sequence $\left(a_{n}\right)_{n \geq 1}$ if $a_{n}$ divides $a_{n+1}$ and if $f \in L^{1}([0,1))$ we have

$$
\lim _{N \rightarrow \infty} \frac{1}{a_{N}} \sum_{j=0}^{a_{N}-1} f\left(\left\{x+\frac{j}{a_{N}}\right\}\right)=\int_{0}^{1} f(t) d t,
$$

almost everywhere with respect to Lebesgue measure.

E.g., $a_{n}=2^{2^{n}}$.

In $1989 \mathrm{~J}$. R os en blatt proved the following theorem.

TheOREM 15. If $\inf _{n \geq 1} \frac{b_{n+1}}{b_{n}}>1$, then given $\delta, \epsilon>0$, and a dynamical system $(X, \beta, \mu, T)$ with $\mu$ non-atomic, there exist $E \in \beta$ with $0<\mu(E)<\epsilon$ such that

$$
\lim _{N \rightarrow \infty} \frac{1}{N} \sum_{n=1}^{N} \chi_{E}\left(T^{b_{n}} x\right) \geq \delta .
$$

Take $T(x)=\{2 x\} \bmod 1$ on $[0,1)$ with $\mu$ equal to Lebesgue measure and $b_{n}=2^{n}$ and we see that $\left(a_{n}\right)_{n \geq 1}$ with $a_{n}=2^{2^{n}}$ is not in $\left(L^{\infty}\right)^{*}$ but satisfies (2) answering Baker's theorem. 


\section{PROBLEMS IN STRONG UNIFORM DISTRIBUTION}

In this context we also have the following obsevation.

Given $p$ in $[1, \infty)$ it is possible to give strictly increasing sequences of integers $\left(c_{k}\right)_{k=1}^{\infty}$ such that $a_{k}=2^{c_{k}}(k=1,2, \ldots)$ is in $\left(L^{p}\right)^{*}$ but not in $\left(L^{q}\right)^{*}$ for any $q<p$. Here $L^{p}$ denotes the space of Lebesgue measurable functions on $[0,1)$ whose $p^{t h}$ powers are Lebesgue integrable. This observation relies on a result of K. Reinhold - L ars on [19].

THEOREM 16. Given $p$ in $[1, \infty)$ there exists a strictly increasing sequence of natural numbers $\left(c_{k}\right)_{k=1}^{\infty}$ such that for every dynamical system $(X, \beta, \mu, T)$ and every function in $L^{p}(X, \beta, \mu)$ there exists $C_{p}>0$ such that if

$$
M f(x)=\sup _{N \geq 1}\left|\frac{1}{N} \sum_{k=1}^{N} f\left(T^{c_{k}} x\right)\right|,
$$

then

where

$$
\mu(\{x \in X: M f(x)>\alpha\}) \leq \frac{C_{p}}{\alpha^{p}}\|f\|_{p}
$$

$$
\|f\|_{p}=\left(\int_{X}|f|^{p}(x) d \mu\right)^{\frac{1}{p}} .
$$

Also if $1<p$, then there exists $f$ in $L^{q}(X, \beta, \mu)$ such that

$$
\lim _{N \rightarrow \infty} \frac{1}{N} \sum_{k=1}^{N} f\left(T^{c_{k}} x\right)
$$

does not have a finite limit for almost all $x$, with respect to $\mu$.

Choosing $X=[0,1), \beta$ to be the Lebesgue $\sigma$-algebra, $\mu$ to be Lebesgue measure and $T x=\{2 x\}$ and using Theorem 16 as before shows that $a_{k}=2^{c_{k}}$ $(k=1,2, \ldots)$ does not belong to $\left(L^{q}\right)^{*}$. To show that $\left(2^{c_{k}}\right)_{k=1}^{\infty}$ is in $\left(L^{p}\right)^{*}$ we need to show that

$$
\lim _{N \rightarrow \infty} \frac{1}{N} \sum_{k=1}^{N} f\left(\left\{2^{c_{k}} x\right\}\right)=\int_{0}^{1} f(t) d t,
$$

almost everywhere with respect to Lebesgue measure. By a classical theorem of $\mathrm{H}$. W e yl [27] this is known for continuous functions on $[0,1)$. Suppose that $\left(f_{n}\right)_{n=1}^{\infty}$ is a sequence of continuous functions on $[0,1)$ converging to $f$ in $L^{p}$ norm. This means that there exists a subsequence $\left(n_{k}\right)_{k=1}^{\infty}$ such that

$$
\sum_{k=1}^{\infty} \int_{0}^{1}\left|f-f_{n_{k}}\right|^{p}(x) d x<\infty .
$$




\section{KWO CHAN - RADHAKRISHNAN NAIR}

This implies that

$$
\sum_{k=1}^{\infty}\left|f-f_{n_{k}}\right|^{p}(x)<\infty
$$

almost everywhere with respect to Lebesgue measure on $[0,1)$. Thus for every $\epsilon>0$, there exists a sequence of functions $\left(f_{\epsilon, k}\right)_{k=1}^{\infty}$ such that

$$
\left\|f-f_{\epsilon, k}\right\|_{p}^{p} \leq \epsilon^{2 k}
$$

and $f_{\epsilon, k}$ tends to $f$ as $k$ tends to infinity almost everywhere with respect to Lebesgue measure on $[0,1)$. Notice that

$$
M(f+g) \leq M(f)+M(g) .
$$

Let

$$
E_{\epsilon, k}:=\left\{x \in[0,1): M\left(f-f_{\epsilon, k}\right)(x)>\epsilon^{\frac{k}{p}}\right\}
$$

and note from Theorem 16 that

$$
\begin{aligned}
\mu\left(E_{\epsilon, k}\right) & \leq C_{p}\left(\frac{1}{\epsilon}\right)^{k} \int_{E_{\epsilon, k}}\left|f-f_{\epsilon, k}\right|^{p}(x) d x \\
& \leq C_{p}\left(\frac{1}{\epsilon}\right)^{k} \epsilon^{2 k}=C_{p} \epsilon^{k} .
\end{aligned}
$$

Let $a_{N}(f, x)$ denote $\frac{1}{N} \sum_{l=1}^{N} f\left(\left\{2^{c_{l}} x\right\}\right)$. Now

This means that

$$
a_{N}(f, x)=a_{N}\left(f-f_{\epsilon, k} x\right)+a_{N}\left(f_{\epsilon, k}, x\right) .
$$

$$
\left|a_{N}(f, x)-\int_{0}^{1} f(t) d t\right| \leq\left|a_{N}\left(f-f_{\epsilon, k}, x\right)\right|+\left|a_{N}\left(f_{\epsilon, k}, x\right)-\int_{0}^{1} f(t) d t\right|
$$

almost everywhere with respect to Lebesgue measure on $[0,1)$. Thus

$$
\limsup _{N \rightarrow \infty}\left|a_{N}(f, x)-\int_{0}^{1} f(t) d t\right| \leq \limsup _{N \rightarrow \infty}\left|a_{N}\left(f-f_{\epsilon, k}, x\right)\right|+\left|\int_{0}^{1}\left(f-f_{\epsilon, k}\right)(t) d t\right|,
$$

which is

$$
\leq M\left(f-f_{\epsilon, k}\right)(x)+\int_{0}^{1}\left|f-f_{\epsilon, k}\right|(t) d t .
$$

Therefore as $N$ tends to infinity we know that $a_{N}(f, x)$ tends to $\int_{0}^{1} f(t) d t$, for all $x$ in $E_{\epsilon}=\cup_{n=1}^{\infty} E_{\epsilon, n}$. Let $B_{\epsilon}$ be the null set off which $f_{\epsilon, k}$ tends to $f$ as $k$ tends to infinity. This means, that

$$
\lambda\left(E_{\epsilon} \cup B_{\epsilon}\right) \leq \sum_{n=1}^{\infty} \lambda\left(E_{\epsilon, k}\right) \leq C_{p} \sum_{k=1}^{\infty} \epsilon^{k}=\frac{C_{p} \epsilon}{1-\epsilon} .
$$

Letting $\epsilon$ tend to zero shows that $\left(2^{c_{k}}\right)_{k=1}^{\infty}$ is $\left(L^{p}\right)^{*}$ for finite $p$. 


\section{PROBLEMS IN STRONG UNIFORM DISTRIBUTION}

\section{REFERENCES}

[1] BAKER, R. C.: Riemann sums and Lebesgue integrals, Q. J. Math. Oxf. II. Ser. 27 (1976), 191-198.

[2] BEWLEY, T.: Extension of the Birkhoff and von Neumann ergodic theorems to semigroup actions, Ann. Inst. Henri Poincaré, Nouv. Sér., Sect. B 7 (1971), 283-291.

[3] BOHL, P.: Über ein der Theorie der säkuären Störungen vorkommendes Problem, J. Reine Angew. Math. 135 (1909), 189-289.

[4] BOURGAIN, J.: On maximal ergodic theory for certain subsets of the integers, Isr. J. Math. 61 (1988), 39-72.

[5] BOURGAIN, J.: Almost sure convergence in ergodic theory, in: Proc. Int. Conf., Almost Everywhere Convergence, Columbus, OH, 1988, Acadamic, Boston, 1989, pp. 145-151.

[6] CHAN, K.-NAIR, R.: A remark on the distribution of Chebychev polynomials on [-1,1], Unif. Distrib. Theory (to appear).

[7] JESSEN, B.: On the approximation of Lebesgue integrals by Riemann sums, Ann. Math. (2) 35 (1934), 248-251.

[8] KHINCHIN, A.: Ein Satz über kettenbrüche arithmetichen anwendungen, Math. Z. 18 (1923), 289-306.

[9] KOKSMA, J. F.-SALEM, R.: Uniform distribution and Lebesgue integration, Acta Sci. Math. (Szeged) 12 (1950); Actual. Math. Centrum, Amsterdam, Rapport ZW 1949, 004, 9 p.

[10] MARSTRAND, J. M.: On Khinchine's conjecture about strong uniform distribution, Proc. Lond. Math. Soc. 21 (1970), 540-556.

[11] NAIR, R.: On strong uniform distribution, Acta Arith. 56 (1990), 183-193.

[12] NAIR, R.: On some arithmetic properties of Lp summable functions, Q. J. Math. Oxf. II. Ser. 47 (1996), 101-105.

[13] NAIR, R.: On strong uniform distribution. II., Monatsh. Math. 132 (2001), 341-348.

[14] NAIR, R.: On a problem of R. C. Baker, Acta Arith. 109 (2003), 343-348.

[15] NAIR, R.: On strong uniform distribution III., Indag. Math. (N.S.) 14 (2003), 233-240.

[16] NAIR, R.: On strong uniform distribution IV., J. Inequal. Appl. 2005, no. 3, 319-327.

[17] NAIR, R.: On polynomial ergodic averages and square functions, in: Number Theory and Polynomials. Proc. of the Workshop (J. McKee et al., eds.), Bristol, UK, 2006, London Math. Soc. Lecture Note Ser., Vol. 352, Cambridge University Press, Cambridge, 2008, pp. 241-254.

[18] RAIKOV, D. A.: On some arithmetic properties of summable functions, Mat. Sb. 1 (43), (1936), 377-384. (In Russian)

[19] REINHOLD-LARSSON, K.: Discrepancy of behaviour of perturbed sequences in $L^{p}$ spaces, Proc. Amer. Math. Soc. 120 (1994), 865-874.

[20] REISZ, F.: Sur la théorie ergodique, Comment. Math. Helv. 17 (1944/45), 221-239.

[21] ROSENBLATT, J.: Universally bad sequences in ergodic theory, in: Almost everywhere convergence II, Proc. 2nd Int. Conf., Evanston/IL (USA) 1989, Academic Press, Boston, MA, 1991, pp. 227-245.

[22] QUAS, A.-WIERDL, M.: Rates of divergence of non-conventional ergodic averages, Ergodic Theory Dynam. Systems 30 (2010), 233-262.

[23] SAWYER, S.: Maximal inequalities of weak type, Ann. of Math. (2) 84 (1966), 157-174. 


\section{KWO CHAN - RADHAKRISHNAN NAIR}

[24] SIERPIŃSKI, W.: On the asymptotic value of a certain sum, Rozprawy Wydz. Mat. Pryzr. Akad. Um. 50 (1910), 1-10. (In Polish)

[25] WALTERS, P.: An Introduction to Ergodic Theory, in: Grad. Texts in Math. Vol. 79, Springer-Verlag, Berlin, 1982.

[26] WEYL, H.: Über der Gibbssche Erscheinung und verwandte Konvergenzphänomenene, Rend. Mat. Palermo 30 (1910), 377-407.

[27] WEYL, H.: Über die Gleichverteilung von Zahlen mod. Eins, Math. Ann. 77 (1916), 313-352.

Received October 6, 2014

Mathematical Sciences

The University of Liverpool

Peach Street

Liverpool L69 7ZL

UNITED KINGDOM

E-mail: nair@liverpool.ac.uk

sgkchan@liverpool.ac.uk 